PROCEEDINGS OF THE

AMERICAN MATHEMATICAL SOCIETY

Volume 137, Number 10, October 2009, Pages 3473-3476

S 0002-9939(09)09914-6

Article electronically published on May 8, 2009

\title{
DEFINING ADDITIVE SUBGROUPS OF THE REALS FROM CONVEX SUBSETS
}

\author{
MICHAEL A. TYCHONIEVICH
}

(Communicated by Julia Knight)

\begin{abstract}
Let $G$ be a subgroup of the additive group of real numbers and let $C \subseteq G$ be infinite and convex in $G$. We show that $G$ is definable in $(\mathbb{R},+, \cdot C)$ and that $\mathbb{Z}$ is definable if $G$ has finite rank. This has a number of consequences for expansions of certain o-minimal structures on the real field by multiplicative groups of complex numbers.
\end{abstract}

We answer some questions about expansions of o-minimal structures on the real field $\overline{\mathbb{R}}:=(\mathbb{R},+, \cdot)$ by various groups. For more detailed treatment of some of the issues raised below, see van den Dries and Günaydın [4] and Miller 6, 7. In this paper, definable means first-order definable with parameters from the appropriate underlying set. Throughout, let $K(=(K,+, \cdot,<))$ be an ordered subfield of $\overline{\mathbb{R}}$, and let $G$ be a nontrivial additive subgroup of $K$.

Proposition 1. Let $C \subseteq G$ be infinite and convex (in $G$ ). Then $G$ is definable in $(K, C)$.

Proof. If $C$ is unbounded, then $G=C \cup(-C) \cup(a+C)$ for some $a \in K$. Suppose now that $C$ is bounded. Then $G$ is not discrete and is thus dense in $K$. By translation and division by some nonzero element of $G$, we reduce to the case that $1 \in G$ and $C=G \cap[-1,1]$. Put $S=\{s \in K: \exists \varepsilon>0, s(C \cap[0, \varepsilon)) \subseteq C\}$. Evidently, $(K, C)$ defines $S$, and $S$ is a subgroup of $(K,+)$ containing 1 . Hence, it suffices to show that $S \cap(0,1) \subseteq C$, for then $G=S+C$. Let $s \in S \cap(0,1)$ and $\varepsilon>0$ be such that $s(C \cap[0, \varepsilon)) \subseteq C$. By density of $G$, there exists $c \in C \cap(0, \varepsilon)$. Note that $s c \in C \subseteq G$. Let $k$ be the smallest positive integer such that $k c>1-\varepsilon$. Then $s k c \in G$ and $1-k c \in C \cap(-\varepsilon, \varepsilon)$, so that $s(1-k c) \in G$ as well. Since $s=s(1-k c)+s k c$, we have $s \in G$ as required.

Proposition 2. If $G$ has finite rank (equivalently, if the $\mathbb{Q}$-linear span of $G$ is finite dimensional) and $C \subseteq G$ is infinite and convex, then $(K, C)$ defines $\mathbb{Z}$.

Proof. By Proposition 1 and division by some nonzero element of $G$, we reduce to the case that $C=G$ and $1 \in G$. Put $R=\{r \in K: r G \subseteq G\}$. Observe that $R$ is a subring of $K$ contained in $G$ and is definable in $(K, G)$. Since $R \subseteq G$, it has finite rank as an additive group. Hence, the fraction field $J$ of $R$ is a finite-degree algebraic extension of $\mathbb{Q}$. By J. Robinson $[10, \mathbb{Z}$ is definable in $(J,+, \cdot)$. Since $J$ is definable in $(K, R)$, so is $\mathbb{Z}$.

Received by the editors October 1, 2008, and, in revised form, December 22, 2008, and February 14,2009

2000 Mathematics Subject Classification. Primary 03C64; Secondary 14P10.

(C)2009 American Mathematical Society 3473

Reverts to public domain 28 years from publication 
Remarks. (i) If $G$ is finitely generated and $1 \in G$, then $(K, G)$ defines $\mathbb{Z}$ for all subrings $K$ of $\mathbb{R}$ containing $G[6,6.1]$. (ii) The set $R$ contains $S$ as in the proof of Proposition 1 equality holds if and only if $G$ is dense. (iii) Of course, undecidability of $\operatorname{Th}(K, C)$ follows, but much more is true when $K=\mathbb{R}$ : every real Borel set is definable in $(\overline{\mathbb{R}}, \mathbb{Z})$. (iv) Any conditions on $G$ that force the field $J$ to be either a finite-degree algebraic extension of $\mathbb{Q}$ or a purely transcendental extension of some $J^{\prime} \subseteq J$ yield definability of $\mathbb{Z}$, again by [10] for the former and by R. Robinson [1] for the latter. A more recent result of Poonen 9 shows that it is sufficient for $J$ to be a finitely generated field extension of $\mathbb{Q}$. (v) If $K$ is real closed and $G$ is a real-closed subfield of $K$, then $(K, G)$ does not define $\mathbb{Z}$ by van den Dries $[3$. We do not know of any $G$ other than real-closed subfields that do not define $\mathbb{Z}$ over $\overline{\mathbb{R}}$.

We now concentrate on the case that $K=\mathbb{R}$ and consider expansions of $\mathbb{R}$ by multiplicative subgroups of $\mathbb{C} \backslash\{0\}$. We make the usual identifications $\mathbb{R} \hookrightarrow \mathbb{C} \cong \mathbb{R}^{2}$. Given $Z \subseteq \mathbb{C}$, let $e^{Z}$ denote the image of $Z$ under complex exponentiation. We focus here on expansions of structures of the form $\left(\overline{\mathbb{R}},\left(e^{w G}\right)_{w \in W}\right)$ where $W \subseteq \mathbb{C}$. At present, there are essentially only two positive results known:

- 6, 7. If $G$ is cyclic and $\mathfrak{R}$ is an o-minimal expansion of $\overline{\mathbb{R}}$ that defines no irrational power functions, then $\left(\mathfrak{R}, e^{G}\right)$ does not define $\mathbb{Z}$. Indeed, every definable set (of any arity) is a boolean combination of open sets (but much more is true). Write $G=\alpha \mathbb{Z}$, put $\omega=2 \pi / \alpha$, and suppose moreover that $\Re$ defines the restriction of the function $t \mapsto \sin (\omega \log t): \mathbb{R}^{>0} \rightarrow \mathbb{R}$ to some nontrivial subinterval of $\mathbb{R}^{>0}$. Then $\left(\mathfrak{R}, e^{G}\right)$ defines the group $e^{(1+i \omega) \mathbb{R}}$. By [2], the expansion of $\overline{\mathbb{R}}$ by the restriction $\sin (\omega \log t) \uparrow[1,2]$ is o-minimal and defines no irrational power functions. This means that $\left(\overline{\mathbb{R}}, e^{i \mathbb{R}}\right)$ does not define $\mathbb{Z}$.

- 4]. If $G$ is noncyclic and has finite rank, then neither $\left(\overline{\mathbb{R}}, e^{G}\right)$ nor $\left(\overline{\mathbb{R}}, e^{i G}\right)$ defines $\mathbb{Z}$. Indeed, every definable set (of any arity) is a boolean combination of $\mathrm{F}_{\sigma}$ sets.

Let $\mathbb{R}_{e}$ and $\mathbb{R}_{s}$ denote the expansions of $\overline{\mathbb{R}}$ by the restrictions exp $\lceil[0,1]$ and $\sin \lceil[0,2 \pi]$ of the real exponential and sine functions. The intervals of definition are picked for convenience: by addition formulas, for any bounded $[a, b] \subseteq \mathbb{R}$ we have $\exp \left\lceil[a, b]\right.$ definable in $\mathbb{R}_{e}$ and $\sin \left\lceil[a, b]\right.$ definable in $\mathbb{R}_{s}$. By $\left[2\right.$, both $\mathbb{R}_{e}$ and $\mathbb{R}_{s}$ are o-minimal and define no irrational power functions (indeed, the same is true of their amalgam). It is worth noting that, by Bianconi [1], $\mathbb{R}_{s}$ does not define exp $\uparrow[0,1]$ and $\mathbb{R}_{e}$ does not define $\sin \lceil[0,2 \pi]$. In his thesis $[5]$, Günaydın shows that for any nonzero $\alpha \in \mathbb{R}$, both $\left(\overline{\mathbb{R}}_{e}, e^{\alpha \mathbb{Q}}\right)$ and $\left(\overline{\mathbb{R}}_{s}, e^{i \alpha \mathbb{Q}}\right)$ define $\mathbb{Q}$ and hence also $\mathbb{Z}$. We now extend his result.

Corollary 3. If $G$ is noncyclic and $C$ is an infinite convex subset of $e^{G}$, then $G$ is definable in $\left(\mathbb{R}_{e}, C\right)$. If moreover $G$ has finite rank, then $\mathbb{Z}$ is definable.

Proof. There exist $0<a<b<\infty$ such that $[a, b] \cap C$ is infinite. As $\log \lceil[a, b]$ is definable in $\mathbb{R}_{e}$, we have $[\log a, \log b] \cap G$ definable in $\left(\mathbb{R}_{e}, C\right)$. Apply Propositions 1$]$ and 2 .

Corollary 4. If $e^{i G}$ is infinite and $A$ is a nondegenerate arc of the unit circle, then $G+2 \pi \mathbb{Z}$ is definable in $\left(\mathbb{R}_{s}, e^{i G} \cap A\right)$. If moreover $G$ has finite rank, then $\mathbb{Z}$ is definable. 
Proof. Intersect $e^{i G} \cap A$ with either the right half-plane or the left half-plane, project onto the second coordinate, and then take the image under arcsin. This set is definable and contains an infinite convex subset of $G+2 \pi \mathbb{Z}$. Apply Proposition 1 . If $G$ has finite rank, then so does $G+2 \pi \mathbb{Z}$. Apply Proposition 2 ,

We have concentrated so far on expanding by single groups, but there are many reasons to be interested in expanding by collections of groups. The most fundamental question is this: For $\mathbb{Q}$-linearly independent $\alpha, \beta \in \mathbb{R}$, what can be said about $\left(\overline{\mathbb{R}}, e^{\alpha \mathbb{Z}}, e^{\beta \mathbb{Z}}\right)$ ? Other than the obvious fact that the direct product $e^{\alpha \mathbb{Z}+\beta \mathbb{Z}}$ is definable, very little known is known as of this writing. The situation is similar for the structures $\left(\overline{\mathbb{R}}, e^{i \alpha \mathbb{Z}}, e^{i \beta \mathbb{Z}}\right)$ and $\left(\overline{\mathbb{R}}, e^{(1+i \alpha) \mathbb{R}}, e^{(1+i \beta) \mathbb{R}}\right)$. We now provide some partial answers.

Corollary 5. If $\alpha, \beta \in \mathbb{R}$ are $\mathbb{Q}$-linearly independent, then $\mathbb{Z}$ is definable in each of the structures

$$
\left(\mathbb{R}_{e}, e^{\alpha \mathbb{Z}}, e^{\beta \mathbb{Z}}\right),\left(\mathbb{R}_{s}, e^{i \alpha \mathbb{Z}}, e^{i \beta \mathbb{Z}}\right),\left(\mathbb{R}_{e}, e^{(1+i \alpha) \mathbb{R}}, e^{(1+i \beta) \mathbb{R}}\right),\left(\mathbb{R}_{s}, e^{(1+i \alpha) \mathbb{R}}, e^{(1+i \beta) \mathbb{R}}\right) .
$$

Proof. Since the product groups are definable, the first two cases are immediate from Corollaries 3 and 4 . For the third, observe that $e^{(1+i \alpha) \mathbb{R}} \cap \mathbb{R}^{>0}$ and $e^{(1+i \beta) \mathbb{R}} \cap$ $\mathbb{R}^{>0}$ are definable, thus reducing to the first case. For the fourth, project $e^{(1+i \alpha) \mathbb{R}} \cap$ $e^{(1+i \beta) \mathbb{R}}$ onto the unit circle and apply Corollary 4 .

As an immediate consequence, we have the following:

Corollary 6. Let $r \in \mathbb{R} \backslash \mathbb{Q}$. Then $\mathbb{Z}$ is definable in each of the structures

$$
\left(\mathbb{R}_{e}, x^{r}, e^{\alpha \mathbb{Z}}\right), \quad\left(\mathbb{R}_{e}, x^{r}, e^{(1+i \alpha) \mathbb{R}}\right), \quad\left(\mathbb{R}_{s}, x^{r}, e^{(1+i \alpha) \mathbb{R}}\right) .
$$

We close with one more application. In [8, Miller and Speissegger establish a trichotomy for expansions of the structure $\mathbb{R}_{\mathrm{an}}$ by individual trajectories of certain kinds of analytic planar vector fields. By combining Corollaries 5 and 6 with Corollary 4 of their paper, the trichotomy extends to expansions of $\mathbb{R}_{\text {an }}$ by arbitrary collections of such trajectories.

\section{ACKNOWLedgement}

I thank my Ph.D. supervisor, Chris Miller, for suggesting the question leading to Proposition 1 and for help in preparing this paper.

\section{REFERENCES}

[1] Ricardo Bianconi, Nondefinability results for expansions of the field of real numbers by the exponential function and by the restricted sine function, J. Symbolic Logic 62 (1997), no. 4, 1173-1178. MR1617985 (99k:03034)

[2] Lou van den Dries, A generalization of the Tarski-Seidenberg theorem, and some nondefinability results, Bull. Amer. Math. Soc. (N.S.) 15 (1986), no. 2, 189-193. MR854552 (88b:03048)

[3] _ Dense pairs of o-minimal structures, Fund. Math. 157 (1998), no. 1, 61-78. MR1623615 (2000a:03058)

[4] Lou van den Dries and Ayhan Günaydın, The fields of real and complex numbers with a small multiplicative group, Proc. London Math. Soc. (3) 93 (2006), no. 1, 43-81. MR2235481 (2007i:03039)

[5] Ayhan Günaydın, Model theory of fields with multiplicative groups, Ph.D. thesis, University of Illinois at Urbana-Champaign, 2008.

[6] Chris Miller, Tameness in expansions of the real field, Logic Colloquium '01, Lect. Notes Log., vol. 20, Assoc. Symbol. Logic, Urbana, IL, 2005, pp. 281-316. MR2143901 (2006j:03049) 
[7] _ Avoiding the projective hierarchy in expansions of the real field by sequences, Proc. Amer. Math. Soc. 134 (2006), no. 5, 1483-1493 (electronic). MR2199196 (2007h:03065)

[8] Chris Miller and Patrick Speissegger, A trichotomy for expansions of $\mathbb{R}_{\text {an }}$ by trajectories of analytic planar vector fields, preliminary report, available at http://www.math.ohio-state. edu/ ${ }^{\sim}$ miller

[9] Bjorn Poonen, Uniform first-order definitions in finitely generated fields, Duke Math. J. 138 (2007), no. 1, 1-22. MR2309154 (2008f:12015)

[10] Julia Robinson, The undecidability of algebraic rings and fields, Proc. Amer. Math. Soc. 10 (1959), 950-957. MR0112842 (22:3691)

[11] Raphael M. Robinson, The undecidability of pure transcendental extensions of real fields, Z. Math. Logik Grundlagen Math. 10 (1964), 275-282. MR0172803 (30:3021)

Department of Mathematics, Ohio State University, 231 West 18th Avenue, ColumBus, Оніо 43210

E-mail address: tycho@math.ohio-state.edu 